\title{
Muscle-Adipose Tissue Cross Talk
}

\author{
Kristin I. Stanford ${ }^{1}$ and Laurie J. Goodyear ${ }^{2,3}$ \\ ${ }^{1}$ Dorothy M. Davis Heart and Lung Research Institute, Department of Physiology and Cell Biology, The Ohio \\ State University Wexner Medical Center, Columbus, Ohio 43210 \\ ${ }^{2}$ Section on Integrative Physiology and Metabolism, Joslin Diabetes Center, Brigham and Women's Hospital, \\ Harvard Medical School, Boston, Massachusetts 02215 \\ ${ }^{3}$ Department of Medicine, Brigham and Women's Hospital, Harvard Medical School, Boston, \\ Massachusetts 02215 \\ Correspondence: laurie.goodyear@joslin.harvard.edu
}

Exercise training results in adaptations to both skeletal muscle and white adipose tissue (WAT) and protects against metabolic disorders including obesity and type 2 diabetes. Exerciseinduced adaptations include an altered profile of secreted proteins, both myokines (from skeletal muscle) and adipokines (from adipose tissue). These secreted proteins may act in an endocrine manner to facilitate tissue-to-tissue communication and "cross talk," likely working together to improve overall metabolic health. Some studies suggest that contracting skeletal muscles release myokines that may function to alter the phenotype of WAT, including WAT "beiging," in which there is increased expression of beige marker genes and increased presence of multilocular cells within the WAT.

$T^{2}$ ansplantation of exercise-trained subcutaneous white adipose tissue (scWAT) into sedentary mice results in improvements in skeletal muscle glucose uptake, suggesting that scWAT acts in an endocrine manner to improve skeletal muscle function. Studies in humans and rodents have shown that both an acute bout of exercise and exercise training can alter adipokines in circulation. Here, we will discuss studies investigating the exerciseinduced regulation of myokines and adipokines and their potential role in skeletal muscle-adipose tissue cross talk. Identification of novel exercise factors could eventually lead to the development of new treatments, including exercise protocols and pharmaceuticals, for the treatment of obesity, type 2 diabetes, and other diseases.

Physical exercise is a well-established tool to prevent, manage, and treat several chronic conditions, including type 2 diabetes, hypertension, heart disease, obesity, and sarcopenia (Haskell et al. 2007; Colberg et al. 2010; Egan and Zierath 2013). Exercise improves metabolic health through adaptations to several tissues, including skeletal muscle and white adipose tissue (WAT). Adaptations to skeletal muscle include improved glucose uptake (Hussey et al. 2012; Howlett et al. 2013; Richter and Hargreaves 2013; Look AHEAD Research Group 2014), increased glucose transporter type 4 (GLUT4) translocation and expression (Higa-

Editors: Juleen R. Zierath, Michael J. Joyner, and John A. Hawley

Additional Perspectives on The Biology of Exercise available at www.perspectivesinmedicine.org

Copyright (C) 2018 Cold Spring Harbor Laboratory Press; all rights reserved; doi: 10.1101/cshperspect.a029801

Cite this article as Cold Spring Harb Perspect Med 2018;8:a029801 
shida et al. 2011; Richter and Hargreaves 2013), augmented mitochondrial activity (Little et al. 2011), increased ability to take up and oxidize fat as a fuel (Jeukendrup 2002), and an increase in exercise-induced myokines into the circulation (Pedersen and Febbraio 2008; Bostrom et al. 2012; Seldin et al. 2012; Rao et al. 2014; Roberts et al. 2014; Dong et al. 2016). Myokines exert autocrine, paracrine, and endocrine effects to influence and regulate whole-body metabolism of glucose and lipids and energy balance and act on WAT, as well as other tissues. Exercise-induced adaptations to WAT include decreased adipocyte size (Craig et al. 1981; Gollisch et al. 2009), reduced lipid content (Craig et al. 1981; Gollisch et al. 2009), increased expression of metabolic proteins such as GLUT4 and peroxisome proliferator-activated receptor$\gamma$ coactivator $1 \alpha$ (PGC-1 $\alpha$ ) (Craig et al. 1981; Hirshman et al. 1989; Stallknecht et al. 1991; Gollisch et al. 2009; Sutherland et al. 2009; Stanford et al. 2015a,b), increased mitochondrial activity (Stallknecht et al. 1991; Stanford et al. 2015a), and altered expression of exercise-induced adipokines (Stanford et al. 2015a,b). Adipokines are secreted factors released from WAT that modulate inflammation, lipid and glucose metabolism, blood pressure, and atherosclerosis (Rabe et al. 2008), and can act in an autocrine, endocrine, or paracrine manner.

Exercise also results in a "beiging" of the subcutaneous WAT (scWAT) (Cao et al. 2011; Bostrom et al. 2012; Rao et al. 2014; Roberts et al. 2014; Trevellin et al. 2014; Stanford et al. 2015a) in rodents, although whether exercise-induced beiging in humans exists is less conclusive (Norheim et al. 2014; Vosselman et al. 2015). "Beiging" indicates the presence of multilocular cells within WAT. These cells are more metabolically active than white adipocytes (Wu et al. 2012) and have a distinct molecular signature, expressing $T b x 1$, Tmem26, and Cd137, gene markers that are not expressed in mature brown or white adipocytes (Cao et al. 2011; Bostrom et al. 2012; Wu et al. 2012; Trevellin et al. 2014; Stanford et al. 2015a). Beige adipoyctes also have increased expression of $U c p 1, \operatorname{Prdm16}$, and other markers of brown adipose tissue (BAT) such as Cidea, Elovl3, Pgc1 $\alpha$, Ppar $\gamma$, Cox8b, Dio2, and Otopetrin.
Although the metabolic improvements to both skeletal muscle and WAT are important in their own right, another important concept in exercise biology is the idea of tissue-to-tissue communication, or "cross talk," which can mediate some of the beneficial effects of exercise. This means that when myokines are released from skeletal muscle in response to exercise, they alter the metabolic function of other tissues, particularly WAT. Adipokines secreted from exercise-trained WAT operate in a similar manner to influence other tissues, specifically to improve the metabolic function of skeletal muscle. Here, we will discuss the exercise-induced release of myokines from skeletal muscle that can alter both the beiging and metabolic function of WAT, and the exercise-induced release of adipokines from WAT that alter skeletal muscle function.

\section{MYOKINES REGULATE THE BEIGING OF scWAT}

Numerous studies have identified various myokines released from skeletal muscle during exercise that can cause a beiging of scWAT (Fig. 1) (Pedersen and Febbraio 2012; Stanford and Goodyear 2016). Exercise-induced myokines include irisin (Bostrom et al. 2012), meteorinlike 1 (Rao et al. 2014), myostatin (Feldman et al. 2006), $\beta$-aminoisobutyric acid (BAIBA) (Roberts et al. 2014), myonectin (Seldin et al. 2012), lactate (Carrière et al. 2014), and fibroblast growth factor 21 (FGF-21) (Lee et al. 2014). In the following sections, we will discuss the potential role of exercise-induced myokines in altering the beiging of scWAT.

Irisin

A recently identified myokine that has been a topic of intense investigation is irisin. Irisin was initially identified through studies in transgenic mice with increased skeletal muscle PGC$1 \alpha$ expression (Bostrom et al. 2012). These mice had beige adipocytes within their scWAT, an effect that was further amplified when mice were exercise trained (Bostrom et al. 2012). Irisin was identified as a polypeptide cleaved from the 
Muscle-Adipose Tissue Cross Talk

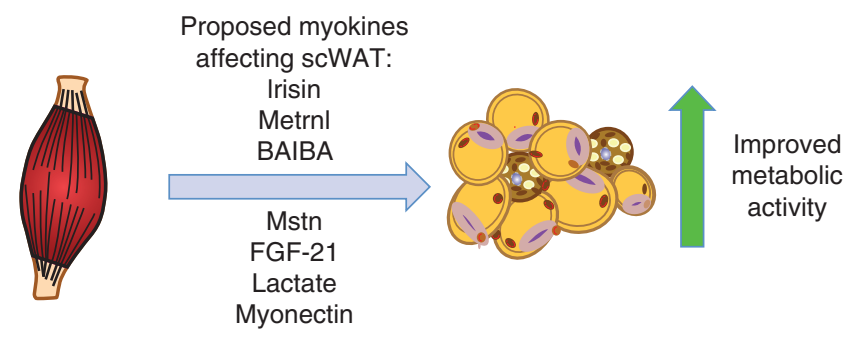

Figure 1. Putative exercise-induced myokines regulating subcutaneous white adipose tissue (scWAT). Multiple studies suggest that exercise causes release of several myokines that may result in beiging and/or improved metabolic activity of scWAT. More studies are needed to understand the mechanism. Metrnl, Meteorin-like; BAIBA, $\beta$-aminoisobutyric acid; Mstn, myostatin; FGF-21, fibroblast growth factor 21.

membrane protein fibronectin type III domaincontaining protein 5 (FNDC5a), and its concentration was significantly increased in circulation after 8 weeks of voluntary wheel-cage running in mice. When mice were injected with an FNDC5-neutralizing antibody, which decreased the concentration of circulating irisin, the exercise-induced beiging of scWAT was reduced. To determine whether circulating irisin was also a factor in human subjects, young healthy males underwent a 10-week endurancetraining program. After 10 weeks, plasma irisin was increased twofold. These data indicate that chronic exercise in both mice and humans significantly increases blood irisin concentrations, and in mice this increase significantly contributes to the beiging of scWAT (Bostrom et al. 2012).

Although these initial data indicate a role for irisin in response to exercise training in both mice and humans, results from several subsequent studies came to varying conclusions (Bostrom et al. 2012; Raschke et al. 2013; Ellefsen et al. 2014; Huh et al. 2014; Norheim et al. 2014; Albrecht et al. 2015; Jedrychowski et al. 2015; Kim et al. 2015). These follow-up studies addressed two specific questions: (1) is circulating irisin increased in humans with exercise, and (2) does an exercise-induced increase in irisin cause a beiging of human scWAT. One study used a combined endurance and strength-training program in male subjects for a 12 -week period and observed no effect on circulating irisin concentrations (Norheim et al. 2014). In addition to the lack of effect on circulating irisin, there was only a modest increase in beiging of scWAT, indicated by increased UCP1 expression. Another study measured circulating irisin after 8 weeks of endurance or strength-training exercise in young men who were overweight or obese. There was no increase in circulating irisin with endurance exercise training, but resistance exercise significantly increased irisin concentrations (Kim et al. 2015). In contrast, a 12-week strength-training program in women ages 20 to 32 did not alter circulating irisin concentrations (Ellefsen et al. 2014). In another study, circulating irisin concentration was measured after both an acute bout of exercise and a 6-week exercisetraining program. Young healthy women preformed a vibration exercise, a moderate intensity exercise that resembles shivering. A single bout of vibration exercise significantly increased circulating irisin ( $\sim$ twofold $)$, but there was no effect of 6 weeks of vibration exercise on serum irisin concentrations (Huh et al. 2014).

It is important to note that a possible reason for the discrepancies in determining the effects of exercise on circulating irisin are the techniques used to measure irisin. Studies have indicated that commercial ELISA kits may not accurately detect irisin (Erickson 2013; Albrecht et al. 2015) because the antibody used measures the transmembrane domain of FNDC5 and not the secreted irisin peptide. The use of these ELISA kits may have resulted in inaccurate measurements of circulating irisin in response to exercise. Recently, studies have begun to use mass spectrometry to quantify the amount of circulating irisin in humans (Daskalopoulou 
et al. 2014; Jedrychowski et al. 2015), allowing for quantification of circulating irisin in an antibody-independent manner. When this technique was used to quantify irisin in human subjects, circulating irisin was increased after both acute exercise (35\% increase; $P<0.001$ ) (Daskalopoulou et al. 2014) and 12 weeks of aerobic interval training (1.2-fold; $P<0.05)$ (Daskalopoulou et al. 2014; Jedrychowski et al. 2015). Thus, studies have shown that both endurance (Bostrom et al. 2012; Daskalopoulou et al. 2014; Jedrychowski et al. 2015) and resistance (Kim et al. 2015) exercise can increase circulating iri$\sin$ in humans. Importantly, the function of the exercise-induced increase in circulating irisin in human subjects is still unknown. Cell culture experiments have indicated that incubation of mouse or rat preadipocytes with irisin increases markers of beiging, including Ucp1 (Bostrom et al. 2012; Shan et al. 2013; Zhang et al. 2014), but when irisin was incubated with isolated human preadipocytes, there was no increase in beiging markers (Schulz et al. 2011). Additionally, several of the studies that measured circulating irisin after exercise in humans did not measure markers of beiging in the scWAT (Bostrom et al. 2012; Daskalopoulou et al. 2014; Jedrychowski et al. 2015; Kim et al. 2015). Thus, more studies are necessary to elucidate the role of irisin as an exercise-induced myokine responsible for the beiging of scWAT in human subjects. As will be discussed below, this interesting molecule has been implicated as a mediator of other putative myokines and their metabolic functions.

\section{Myostatin}

Myostatin is a myokine that inhibits skeletal muscle cell growth and differentiation (McPherron et al. 1997; McNally 2004). Myostatin knockout mice have an increased muscle mass and decreased fat mass (Whittemore et al. 2003), as well as a dramatic increase in beiging of scWAT (Shan et al. 2013). Cells isolated from the stromal vascular fraction (SVF) of scWAT and incubated with conditioned media from the skeletal muscle of myostatin knockout mice have a marked increase in beiging of the
SVF cells, suggesting a role for myostatin as a muscle myokine that inhibits the beiging of scWAT (Shan et al. 2013; Dong et al. 2016).

\section{Meteorin-Like (Metrnl)}

The myokine Metrnl was identified from a screen analyzing factors secreted from primary myotubes overexpressing PGC- $1 \alpha 4$ (Rao et al. 2014), the splice form of PGC- $1 \alpha$ that regulates skeletal muscle hypertrophy and energy expenditure (Ruas et al. 2012). Mice that received injections of adenoviral vectors containing Metrnl, resulting in a fivefold to sixfold increase in circulating Metrnl, had a significant increase in whole-body energy expenditure. The increase in whole-body energy expenditure was correlated with an increased beiging of scWAT (Rao et al. 2014). Importantly, administration of an anti-Metrnl antibody partially prevented cold-induced beiging of scWAT, indicating that Metrnl is likely an important myokine mediating the beiging phenotype. Exercise also increased Metrnl expression in mice and humans; a single bout of eccentric exercise (downhill running) increased Metrnl messenger RNA (mRNA) expression by threefold in triceps muscle and in circulation in mice, whereas a single bout of combined resistance and aerobic exercise significantly increased circulating Metrnl in humans. Interestingly, voluntary wheel-cage exercise in mice, which is a well-established training method to induce beiging of scWAT, did not increase Metrnl expression. These data suggest that Metrnl functions in the beiging of scWAT and is increased in response to resistance exercise in mice and humans. The role of Metrnl in exercise-induced beiging in human scWAT requires future investigation.

\section{$\beta$-Aminoisobutyric Acid}

BAIBA (Roberts et al. 2014) is another myokine that has been recently identified to have a role in the beiging of scWAT after exercise. BAIBA was identified as a potential myokine by liquid chromatography-mass spectrometry (LC-MS) metabolic profiling of human myocytes that overexpressed PGC- $1 \alpha$. The addition of BAIBA 
to the drinking water of mice significantly increased the expression of the beiging genes $U_{c p} 1$ and Cidea in scWAT, and the incubation of primary SVF isolated from WAT with BAIBA increased the expression of Ucp1 and Cidea in the SVF. Human pluripotent stem cells that were exposed to BAIBA while undergoing differentiation to mature white adipocytes had an increased expression of the beiging markers. Exercise significantly increased BAIBA expression in both mice and humans. In mice, 3 weeks of voluntary wheel-cage running significantly increased circulating BAIBA, whereas in humans, 20 weeks of supervised submaximal aerobic exercise training (HERITAGE Family Study) in 80 subjects resulted in a significant increase in circulating BAIBA (Roberts et al. 2014). These data suggest that exercise in both mice and human subjects significantly increased circulating BAIBA and, in rodents and isolated human cells, BAIBA may play a role in increased beiging of scWAT. Similar to the myokines Metrnl and irisin, the direct role of BAIBA in exercise-induced beiging in human scWAT has yet to be established.

\section{Fibroblast Growth Factor 21}

FGF-21 is a member of the FGF superfamily that is involved in the regulation of systemic glucose and lipid metabolism (Kharitonenkov et al. 2005; Kharitonenkov and Larsen 2011) as well as the beiging of scWAT in response to cold exposure (Hondares et al. 2011). Although the role of FGF-21 in response to exercise has not been fully established, recent studies have shown that an exercise-induced increase in irisin can stimulate FGF-21, resulting in increased beiging of scWAT in both human and mouse adipocytes (Lee et al. 2014).

\section{Lactate}

Lactate has also been recently shown to induce beiging of both human and mouse white adipocytes and scWAT. Lactate is the end product of anaerobic glycolysis and is typically considered a metabolic waste product. Intense exercise causes a $\sim 20$-fold increase in circulating lactate.
Lactate was recently identified as a signaling molecule derived from muscle to communicate with other tissues, particularly the brain, the liver, and the heart (Brooks 2009). To determine whether lactate, such as the amount that would accumulate after either exercise or cold exposure, could also act as a myokine that altered scWAT, mice were placed at $4^{\circ} \mathrm{C}$ for 1 to 7 days. After cold exposure, mice had an increase in beiging and Ucp1 expression in scWAT, and the increase in beiging correlated with the increase in circulating lactate and expression of the lactate-importing transporter Mct1 in scWAT (Carrière et al. 2014).

To directly test the effects of lactate on the beiging of scWAT, human and mouse white adipocytes were incubated with lactate. After $24 \mathrm{~h}$, the beiging genes Ucp1, Cidea, and Fgf21 were significantly increased. To determine whether the lactate-induced beiging also occurred in vivo, rosiglitazone-treated mice were injected intraperitoneally with lactate every day for 11 days. These lactate injections enhanced Ucp1 and Cidea expression in the scWAT (Carrière et al. 2014). These data indicate that an increase in lactate can have a direct effect on the beiging of scWAT. To this point, the effects of an exerciseinduced increase in lactate to increase expression of Ucp1 and MCT1 have only been studied in BAT (De Matteis et al. 2013), and future investigations are needed to directly link an exercise-induced increase in lactate to the remodeling and beiging of scWAT.

\section{MYOKINES THAT IMPROVE WAT METABOLISM THROUGH MECHANISMS OTHER THAN BEIGING}

Another myokine, myonectin, has been identified to improve glucose and lipid metabolism in scWAT. Myonectin, or $\mathrm{Cl} / \mathrm{q} /$ tumor necrosis factor (TNF)-related protein isoform 15 (CTRP15), was discovered as a myokine that functions to improve lipid metabolism in scWAT. Myonectin is regulated by both diet and exercise because it is decreased in response to fasting, increased after feeding, and increased after chronic exercise training (Seldin et al. 2012). Two weeks of voluntary exercise (wheel- 
cage running) in mice significantly increased myonectin expression in the soleus and plantaris muscles, as well as in circulation. It is not clear if myonectin increases in concentration in response to the exercise itself, or to the refeeding that occurs after exercise. Myonectin functions to decrease plasma-free fatty acids (FFAs) by increasing CD36 and FATP1 in adipose tissue, which stimulates adipose tissue FFA uptake. These effects appear to be specific to fatty acid uptake because administration of exogenous myonectin decreases serum FFA but has no effect on adipose tissue lipolysis or glucose metabolism (Seldin et al. 2012).

\section{DOES EXERCISE-TRAINED ADIPOSE TISSUE REGULATE SKELETAL MUSCLE FUNCTION?}

Exercise training increases metabolic activity and mitochondrial function of adipose tissue, alters adipokine expression within adipose tissue, and affects circulating concentrations of adipokines. Although these training-induced adaptations are observed in both perigonadal (pg)WAT and scWAT, they are more pronounced in scWAT. Previous work in our laboratory investigated if these exercise-induced adaptations to WAT could improve whole-body metabolic health. To examine this, sedentary recipient mice were transplanted with scWAT from donor mice trained by voluntary wheel running for 11 days (Stanford et al. 2015a). Control mice were either transplanted with scWAT from sedentary mice or sham operated. Nine days after transplantation, there was a significant improvement in glucose tolerance in mice transplanted with scWAT from trained mice compared with both shamoperated mice and mice transplanted with scWAT from sedentary mice. This marked improvement in glucose tolerance was accompanied by decreased fasting levels of blood glucose, insulin, and cholesterol. This improvement was short-lived; there was no difference in glucose tolerance in mice that received trained or sedentary scWAT 28 days posttransplantation. There were also no differences in body weight, food intake, energy expenditure, or spontaneous activity observed among groups throughout the 28 days posttransplantation.

Interestingly, there was no improvement in glucose tolerance when trained pgWAT was transplanted into recipient mice, indicating that there is a specific exercise-induced adaptation to scWAT that results in improved metabolic health in recipient mice.

To determine which tissue was most responsible for the increase in glucose uptake in the mice that were transplanted with trained scWAT, we measured insulin-stimulated glucose disposal in vivo. These data reveal increased rates of glucose uptake into oxidative skeletal muscles and BAT in mice transplanted with trained scWAT. These exciting data indicate that transplantation of scWAT from trained mice improves skeletal muscle glucose uptake, likely through an endocrine-related mechanism (Stanford et al. 2015a). These endocrine effects are likely mediated by the release of adipokines that are unique to the trained scWAT. Although the specific adipokine(s) secreted from trained scWAT that could influence skeletal muscle glucose uptake have not been identified, microarray analysis revealed several putative secreted proteins that were significantly increased in scWAT from trained mice (Stanford et al. 2015a). These data suggest that in addition to exercise triggering skeletal muscle to release myokines and result in the stimulation of tissue-to-tissue cross talk, exercise also stimulates adipose tissue to release adipokines, which may improve skeletal muscle and systemic metabolism.

\section{DO EXERCISE-INDUCED ADIPOKINES IMPROVE SKELETAL MUSCLE FUNCTION?}

Several studies in humans and rodents have investigated the effects of exercise training on adipokine expression and secretion, particularly the two most well-studied adipokines, leptin and adiponectin (Zachwieja et al. 1997; Kraemer et al. 1999; Kanaley et al. 2001; Hauner 2005; Bradley et al. 2008; Halberg et al. 2008; Bouassida et al. 2010; Golbidi and Laher 2014; Zhang et al. 2014), and the cytokine TNF- $\alpha$ (Klimcakova et al. 2006; Polak et al. 2006; Clark 
2015). Leptin is secreted by adipocytes and helps to regulate energy balance by acting as an appetite suppressant. The amount of leptin in circulation is correlated with adiposity. Because exercise training reduces adiposity, exercise results in decreased circulating leptin in both rodents and humans (Zachwieja et al. 1997; Kraemer et al. 1999; Kanaley et al. 2001; Bradley et al. 2008; Bouassida et al. 2010; Golbidi and Laher 2014). The concentration of adiponectin in circulation is inversely correlated to fat mass and is linked to increased insulin sensitivity (Berg et al. 2002; Kim et al. 2007). TNF$\alpha$ is increased in adipose tissue during obesity and can alter the endocrine functions of adipose tissue (Cawthorn and Sethi 2008).

A recent study performed a meta-analysis of 66 publications comparing several combinations of exercise and dietary interventions in healthy adults aged 18 to 65 (Clark 2015). This analysis examined studies that used dietary interventions in combination with endurance or resistance exercise over at least a 4-week period. Each of these studies revealed striking improvements in body mass, circulating blood hormones, and circulating lipids in response to an exercise intervention. Both resistance and endurance exercise interventions elicited positive changes in the adipokine profile by increasing adiponectin concentrations and decreasing both leptin and TNF- $\alpha$ in circulation. These data indicate that either type of exercise combined with diet intervention significantly improves circulating adipokines.

In contrast to these data, studies in obese human subjects showed that a 12-week exercise-training program resulted in little or no change in adipokine mRNA for LEP, ADIPOQ, $I L-6$, or TNF- $\alpha$ in scWAT (Klimcakova et al. 2006; Polak et al. 2006). Serum concentrations of adipokines were not measured in these studies, so it is not clear what the effect of exercise in obese subjects is on circulating adipokines. Taken together, these data indicate that exercise in healthy adults improves the adipokine profile. However, the role of exercise-induced changes in adipokines on skeletal muscle metabolism, as well as how they change in response to exercise in obese patients, is unclear.
There is less known about the effects of exercise on the circulating adipokine retinol-binding protein 4 (RBP4). Serum RBP4 is increased in both mouse and human insulin resistance. One study examined the effects of exercise training on RBP4 in insulin-resistant human subjects. These subjects underwent supervised exercise training ( $60 \mathrm{~min}$ of bicycling and running per day, 3 days a week) for 4 weeks. At the end of the training period, euglycemic-hyperinsulinemic clamps were performed to determine insulin sensitivity. Circulating RBP4 was significantly decreased in human subjects who had improved insulin sensitivity after the exercise regimen. However, subjects whose insulin sensitivity did not improve in response to exercise training had no decrease in circulating RBP4 (Graham et al. 2006). These data suggest that exercise-induced improvements in insulin resistance are inversely correlated to circulating RBP4, although further studies are needed to truly define the role of exercise on RBP4. Taken together, these studies indicate that exercise alters the adipokine profile, but the direction of these changes and their effects on metabolism will be a topic of future investigation.

The physiological consequence of the exercise-induced regulation of adipokines, as well as the mechanism of exercise-induced adipokine regulation, is a topic of intense investigation. It is possible that increased expression of adipokines enhances free fatty acid supply to working skeletal muscle (Zachwieja et al. 1997; Bradley et al. 2008; Halberg et al. 2008), or that the altered adipokine profile contributes to regulation of skeletal muscle or whole-body glucose homeostasis.

\section{CONCLUSION}

Exercise training results in profound changes to skeletal muscle and WAT, including an altered profile of both myokines and adipokines. These secreted proteins act in an endocrine manner to facilitate tissue-to-tissue communication and "cross talk," likely working together to improve overall metabolic health. Although investigations of exercise-induced myokines to improve the metabolic profile and increase beiging in 
WAT have been well established in rodents, future studies are needed to determine the mechanisms through which adipokines improve skeletal muscle function. Given the increasing prevalence of obesity and type 2 diabetes, human exercise studies will be critical to gain further insight into the function of novel adipokines, and define their role in skeletal muscle and whole-body metabolism, as well as their impact on human health. Exercise-induced adipokines may have additional benefits on overall health beyond glucose metabolism and could be interesting novel therapeutic targets for obesity, type 2 diabetes, and other diseases.

\section{ACKNOWLEDGMENTS}

This work is supported by National Institutes of Health Grants K01-DK-105109 (to K.I.S.), R01DK112283 and R01-DK099511 (to L.J.G.), and 5P30 DK36836 (Joslin Diabetes Center Diabetes Research Center).

\section{REFERENCES}

Albrecht E, Norheim F, Thiede B, Holen T, Ohashi T, Schering L, Lee S, Brenmoehl J, Thomas S, Drevon CA, et al. 2015. Irisin-A myth rather than an exercise-inducible myokine. Sci Rep 5: 8889.

Berg AH, Combs TP, Scherer PE. 2002. ACRP30/adiponectin: An adipokine regulating glucose and lipid metabolism. Trends Endocrinol Metab 13: 84-89.

Bostrom P, Boström P, Wu J, Jedrychowski MP, Korde A, Ye L, Lo JC, Rasbach KA, Boström EA, Choi JH, et al. 2012. A PGC1- $\alpha$-dependent myokine that drives brown-fatlike development of white fat and thermogenesis. Nature 481: 463-468.

Bouassida A, Chamari K, Zaouali M, Feki Y, Zbidi A, Tabka Z. 2010. Review on leptin and adiponectin responses and adaptations to acute and chronic exercise. Br J Sports Med 44: $620-630$.

Bradley RL, Jeon JY, Liu FF, Maratos-Flier E. 2008. Voluntary exercise improves insulin sensitivity and adipose tissue inflammation in diet-induced obese mice. Am J Physiol Endocrinol Metab 295: E586-E594.

Brooks GA. 2009. Cell-cell and intracellular lactate shuttles. J Physiol 587: 5591-5600.

Cao L, Choi EY, Liu X, Martin A, Wang C, Xu X, During MJ. 2011. White to brown fat phenotypic switch induced by genetic and environmental activation of a hypothalamicadipocyte axis. Cell Metab 14: 324-338.

Carrière A, Jeanson Y, Berger-Müller S, André M, Chenouard V, Arnaud E, Barreau C, Walther R, Galinier A, Wdziekonski B, et al. 2014. Browning of white adipose cells by intermediate metabolites: An adaptive mechanism to alleviate redox pressure. Diabetes 63: 3253-3265.

Cawthorn WP, Sethi JK. 2008. TNF- $\alpha$ and adipocyte biology. FEBS Lett 582: 117-131.

Clark JE. 2015. Diet, exercise or diet with exercise: Comparing the effectiveness of treatment options for weight-loss and changes in fitness for adults (18-65 years old) who are overfat, or obese; systematic review and meta-analysis. J Diabetes Metab Disord 14: 31.

Colberg SR, Albright AL, Blissmer BJ, Braun B, ChasanTaber L, Fernhall B, Regensteiner JG, Rubin RR, Sigal RJ; American College of Sports Medicine; American Diabetes Association. 2010. Exercise and type 2 diabetes: American College of Sports Medicine and the American Diabetes Association: Joint position statement. Exercise and type 2 diabetes. Med Sci Sports Exerc 42: 2282-2303.

Craig BW, Hammons GT, Garthwaite SM, Jarett L, Holloszy JO. 1981. Adaptation of fat cells to exercise: Response of glucose uptake and oxidation to insulin. J Appl Physiol Respir Environ Exerc Physiol 51: 1500-1506.

Daskalopoulou SS, Cooke AB, Gomez YH, Mutter AF, Filippaios A, Mesfum ET, Mantzoros CS. 2014. Plasma irisin levels progressively increase in response to increasing exercise workloads in young, healthy, active subjects. Eur J Endocrinol 171: 343-352.

De Matteis R, Lucertini F, Guescini M, Polidori E, Zeppa S, Stocchi V, Cinti S, Cuppini R. 2013. Exercise as a new physiological stimulus for brown adipose tissue activity. Nutr Metab Cardiovasc Dis 23: 582-590.

Dong J, Dong Y, Dong Y, Chen F, Mitch WE, Zhang L. 2016 Inhibition of myostatin in mice improves insulin sensitivity via irisin-mediated cross talk between muscle and adipose tissues. Int J Obes (Lond) 40: 434-442.

Egan B, Zierath JR. 2013. Exercise metabolism and the molecular regulation of skeletal muscle adaptation. Cell Metab 17: 162-184.

Ellefsen S, Vikmoen O, Slettaløkken G, Whist JE, Nygaard H, Hollan I, Rauk I, Vegge G, Strand TA, Raastad T, et al. 2014. Irisin and FNDC5: Effects of 12-week strength training, and relations to muscle phenotype and body mass composition in untrained women. Eur J Appl Physiol 114: 1875-1888.

Erickson HP. 2013. Irisin and FNDC5 in retrospect: An exercise hormone or a transmembrane receptor? Adipocyte 2: 289-293.

Feldman BJ, Streeper RS, Farese RVJr, Yamamoto KR. 2006. Myostatin modulates adipogenesis to generate adipocytes with favorable metabolic effects. Proc Natl Acad Sci 103: 15675-15680.

Golbidi S, Laher I. 2014. Exercise induced adipokine changes and the metabolic syndrome. J Diabetes Res 2014: 726861.

Gollisch KS, Brandauer J, Jessen N, Toyoda T, Nayer A, Hirshman MF, Goodyear LJ. 2009. Effects of exercise training on subcutaneous and visceral adipose tissue in normal- and high-fat diet-fed rats. Am J Physiol Endocrinol Metab 297: E495-E504.

Graham TE, Yang Q, Blüher M, Hammarstedt A, Ciaraldi TP, Henry RR, Wason CJ, Oberbach A, Jansson PA, Smith $\mathrm{U}$, et al. 2006. Retinol-binding protein 4 and insulin resistance in lean, obese, and diabetic subjects. NEngl J Med 354: 2552-2563. 
Halberg N, Wernstedt-Asterholm I, Scherer PE. 2008. The adipocyte as an endocrine cell. Endocrinol Metab Clin North Am 37: 753-768.

Haskell WL, Lee IM, Pate RR, Powell KE, Blair SN, Franklin BA, Macera CA, Heath GW, Thompson PD, Bauman A. 2007. Physical activity and public health: Updated recommendation for adults from the American College of Sports Medicine and the American Heart Association. Med Sci Sports Exerc 39: 1423-1434.

Hauner H. 2005. Secretory factors from human adipose tissue and their functional role. Proc Nutr Soc 64: 163 169.

Higashida K, Kim SH, Higuchi M, Holloszy JO, Han DH. 2011. Normal adaptations to exercise despite protection against oxidative stress. Am J Physiol Endocrinol Metab 301: E779-E784.

Hirshman MF, Wardzala LJ, Goodyear LJ, Fuller SP, Horton ED, Horton ES. 1989. Exercise training increases the number of glucose transporters in rat adipose cells. Am J Physiol 257: E520-E530.

Hondares E, Iglesias R, Giralt A, Gonzalez FJ, Giralt M, Mampel T, Villarroya F. 2011. Thermogenic activation induces FGF21 expression and release in brown adipose tissue. J Biol Chem 286: 12983-12990.

Howlett KF, Andrikopoulos S, Proietto J, Hargreaves M. 2013. Exercise-induced muscle glucose uptake in mice with graded, muscle-specific GLUT-4 deletion. Physiol Rep 1: e00065.

Huh JY, Mougios V, Skraparlis A, Kabasakalis A, Mantzoros CS. 2014. Irisin in response to acute and chronic wholebody vibration exercise in humans. Metabolism 63: 918 921.

Hussey SE, McGee SL, Garnham A, McConell GK, Hargreaves M. 2012. Exercise increases skeletal muscle GLUT4 gene expression in patients with type 2 diabetes. Diabetes Obes Metab 14: 768-771.

Jedrychowski MP, Wrann CD, Paulo JA, Gerber KK, Szpyt J, Robinson MM, Nair KS, Gygi SP, Spiegelman BM. 2015 Detection and quantitation of circulating human irisin by tandem mass spectrometry. Cell Metab 22: 734-740.

Jeukendrup AE. 2002. Regulation of fat metabolism in skeletal muscle. Ann NY Acad Sci 967: 217-223.

Kanaley JA, Fenicchia LM, Miller CS, Ploutz-Synder LL, Weinstock RS, Carhart R, Azevedo JLJr. 2001. Resting leptin responses to acute and chronic resistance training in type 2 diabetic men and women. Int J Obes Relat Metab Disord 25: 1474-1480.

Kharitonenkov A, Larsen P. 2011. FGF21 reloaded: Challenges of a rapidly growing field. Trends Endocrinol Metab 22: $81-86$.

Kharitonenkov A, Shiyanova TL, Koester A, Ford AM, Micanovic R, Galbreath EJ, Sandusky GE, Hammond LJ, Moyers JS, Owens RA, et al. 2005. FGF-21 as a novel metabolic regulator. J Clin Invest 115: 1627-1635.

Kim JY, van de Wall E, Laplante M, Azzara A, Trujillo ME, Hofmann SM, Schraw T, Durand JL, Li H, Li G, et al. 2007. Obesity-associated improvements in metabolic profile through expansion of adipose tissue. J Clin Invest 117: 2621-2637.

Kim H, Lee HJ, So B, Son JS, Yoon D, Song W. 2015. Effect of aerobic training and resistance training on circulating irisin level and their association with change of body composition in overweight/obese adults: A pilot study. Physiol Res 65: 271-279.

Klimcakova E, Polak J, Moro C, Hejnova J, Majercik M, Viguerie N, Berlan M, Langin D, Stich V. 2006. Dynamic strength training improves insulin sensitivity without altering plasma levels and gene expression of adipokines in subcutaneous adipose tissue in obese men. J Clin Endocrinol Metab 91: 5107-5112.

Kraemer RR, Johnson LG, Haltom R, Kraemer GR, Hebert EP, Gimpel T, Castracane VD. 1999. Serum leptin concentrations in response to acute exercise in postmenopausal women with and without hormone replacement therapy. Proc Soc Exp Biol Med 221: 171-177.

Lee P, Linderman JD, Smith S, Brychta RJ, Wang J, Idelson C, Perron RM, Werner CD, Phan GQ, Kammula US, et al. 2014. Irisin and FGF21 are cold-induced endocrine activators of brown fat function in humans. Cell Metab 19: 302-309.

Little JP, Gillen JB, Percival ME, Safdar A, Tarnopolsky MA, Punthakee Z, Jung ME, Gibala MJ. 2011. Low-volume high-intensity interval training reduces hyperglycemia and increases muscle mitochondrial capacity in patients with type 2 diabetes. J Appl Physiol (1985) 111: $1554-$ 1560.

Look AHEAD Research Group. 2014. Effect of a long-term behavioural weight loss intervention on nephropathy in overweight or obese adults with type 2 diabetes: A secondary analysis of the Look AHEAD randomised clinical trial. Lancet Diabetes Endocrinol 2: 801-809.

McNally EM. 2004. Powerful genes-Myostatin regulation of human muscle mass. N Engl J Med 350: 2642-2644.

McPherron AC, Lawler AM, Lee SJ. 1997. Regulation of skeletal muscle mass in mice by a new TGF- $\beta$ superfamily member. Nature 387: 83-90.

Norheim F, Langleite TM, Hjorth M, Holen T, Kielland A, Stadheim HK, Gulseth HL, Birkeland KI, Jensen J, Drevon CA. 2014. The effects of acute and chronic exercise on PGC- $1 \alpha$, irisin and browning of subcutaneous adipose tissue in humans. FEBS J 281: 739-749.

Pedersen BK, Febbraio MA. 2008. Muscle as an endocrine organ: Focus on muscle-derived interleukin-6. Physiol Rev 88: 1379-1406.

Pedersen BK, Febbraio MA. 2012. Muscles, exercise and obesity: Skeletal muscle as a secretory organ. Nat Rev Endocrinol 8: 457-465.

Polak J, Klimcakova E, Moro C, Viguerie N, Berlan M, Hejnova J, Richterova B, Kraus I, Langin D, Stich V. 2006. Effect of aerobic training on plasma levels and subcutaneous abdominal adipose tissue gene expression of adiponectin, leptin, interleukin 6 , and tumor necrosis factor $\alpha$ in obese women. Metabolism 55: 1375-1381.

Rabe K, Lehrke M, Parhofer KG, Broedl UC. 2008. Adipokines and insulin resistance. Mol Med 14: 741-751.

Rao RR, Long JZ, White JP, Svensson KJ, Lou J, Lokurkar I, Jedrychowski MP, Ruas JL, Wrann CD, Lo JC, et al. 2014. Meteorin-like is a hormone that regulates immune-adipose interactions to increase beige fat thermogenesis. Cell 157: $1279-1291$.

Raschke S, Elsen M, Gassenhuber $H$, Sommerfeld $M$, Schwahn U, Brockmann B, Jung R, Wisløff U, Tjønna 
K.I. Stanford and L.J. Goodyear

AE, Raastad T, et al. 2013. Evidence against a beneficial effect of irisin in humans. PLOS ONE 8: e73680.

Richter EA, Hargreaves M. 2013. Exercise, GLUT4, and skeletal muscle glucose uptake. Physiol Rev 93: 993-1017.

Roberts LD, Boström P, O’Sullivan JF, Schinzel RT, Lewis GD, Dejam A, Lee YK, Palma MJ, Calhoun S, Georgiadi A, et al. 2014. $\beta$-Aminoisobutyric acid induces browning of white fat and hepatic $\beta$-oxidation and is inversely correlated with cardiometabolic risk factors. Cell Metab 19: 96-108.

Ruas JL, White JP, Rao RR, Kleiner S, Brannan KT, Harrison BC, Greene NP, Wu J, Estall JL, Irving BA, et al. 2012. A PGC- $1 \alpha$ isoform induced by resistance training regulates skeletal muscle hypertrophy. Cell 151: 1319-1331.

Schulz TJ, Huang TL, Tran TT, Zhang H, Townsend KL, Shadrach JL, Cerletti M, McDougall LE, Giorgadze N, Tchkonia T, et al. 2011. Identification of inducible brown adipocyte progenitors residing in skeletal muscle and white fat. Proc Natl Acad Sci 108: 143-148.

Seldin MM, Peterson JM, Byerly MS, Wei Z, Wong GW 2012. Myonectin (CTRP15), a novel myokine that links skeletal muscle to systemic lipid homeostasis. J Biol Chem 287: 11968-11980.

Shan T, Liang X, Bi P, Kuang S. 2013. Myostatin knockout drives browning of white adipose tissue through activating the AMPK-PGC1 $\alpha$-Fndc5 pathway in muscle. FASEB J 27: $1981-1989$.

Stallknecht B, Vinten J, Ploug T, Galbo H. 1991. Increased activities of mitochondrial enzymes in white adipose tissue in trained rats. Am J Physiol 261: E410-E414.

Stanford KI, Goodyear LJ. 2016. Exercise regulation of adipose tissue. Adipocyte 5: 153-162.

Stanford KI, Middelbeek RJ, Townsend KL, Lee MY, Takahashi $\mathrm{H}$, So K, Hitchcox KM, Markan KR, Hellbach K, Hirshman MF, et al. 2015a. A novel role for subcutaneous adipose tissue in exercise-induced improvements in glucose homeostasis. Diabetes 64: 2002-2014.
Stanford KI, Middelbeek RJ, Goodyear LJ. 2015b. Exercise effects on white adipose tissue: Beiging and metabolic adaptations. Diabetes 64: 2361-2368.

Sutherland LN, Bomhof MR, Capozzi LC, Basaraba SA, Wright DC. 2009. Exercise and adrenaline increase PGC- $1 \alpha$ mRNA expression in rat adipose tissue. J Physiol 587: $1607-1617$.

Trevellin E, Scorzeto M, Olivieri M, Granzotto M, Valerio A, Tedesco L, Fabris R, Serra R, Quarta M, Reggiani C, et al. 2014. Exercise training induces mitochondrial biogenesis and glucose uptake in subcutaneous adipose tissue through eNOS-dependent mechanisms. Diabetes 63: 2800-2811.

Vosselman MJ, Hoeks J, Brans B, Pallubinsky H, Nascimento EB, van der Lans AA, Broeders EP, Mottaghy FM, Schrauwen P, van Marken Lichtenbelt WD. 2015. Low brown adipose tissue activity in endurance-trained compared with lean sedentary men. Int J Obesity (Lond) 39: 1696-1702.

Whittemore LA, Song K, Li X, Aghajanian J, Davies M, Girgenrath S, Hill JJ, Jalenak M, Kelley P, Knight A, et al. 2003. Inhibition of myostatin in adult mice increases skeletal muscle mass and strength. Biochem Biophys Res Commun 300: 965-971.

Wu J, Boström P, Sparks LM, Ye L, Choi JH, Giang AH, Khandekar M, Virtanen KA, Nuutila P, Schaart G, et al. 2012. Beige adipocytes are a distinct type of thermogenic fat cell in mouse and human. Cell 150: 366-376.

Zachwieja JJ, Hendry SL, Smith SR, Harris RB. 1997. Voluntary wheel running decreases adipose tissue mass and expression of leptin mRNA in Osborne-Mendel rats. Diabetes 46: 1159-1166.

Zhang Y, Li R, Meng Y, Li S, Donelan W, Zhao Y, Qi L, Zhang M, Wang X, Cui T, et al. 2014. Irisin stimulates browning of white adipocytes through mitogen-activated protein kinase p38 MAP kinase and ERK MAP kinase signaling. Diabetes 63: 514-525. 


\section{$\&_{\mathrm{CSH}}^{\infty} \&$ Cold Spring Harbor

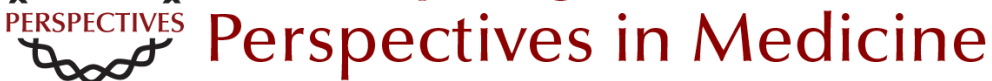

\section{Muscle-Adipose Tissue Cross Talk}

Kristin I. Stanford and Laurie J. Goodyear

Cold Spring Harb Perspect Med 2018; doi: 10.1101/cshperspect.a029801 originally published online May 15, 2017

\section{Subject Collection The Biology of Exercise}

Exosomes as Mediators of the Systemic

Adaptations to Endurance Exercise Adeel Safdar and Mark A. Tarnopolsky

Molecular Basis of Exercise-Induced Skeletal

Muscle Mitochondrial Biogenesis: Historical

Advances, Current Knowledge, and Future

Challenges

Christopher G.R. Perry and John A. Hawley

Exercise Metabolism: Fuels for the Fire Mark Hargreaves and Lawrence L. Spriet

Health Benefits of Exercise Gregory N. Ruegsegger and Frank W. Booth

Molecular Regulation of Exercise-Induced Muscle

Fiber Hypertrophy

Marcas M. Bamman, Brandon M. Roberts and Gregory R. Adams

Physiological Redundancy and the Integrative

Responses to Exercise Michael J. Joyner and Jerome A. Dempsey

On the Run for Hippocampal Plasticity C'iana Cooper, Hyo Youl Moon and Henriette van Praag

Molecular Basis for Exercise-Induced Fatigue: The Importance of Strictly Controlled Cellular $\mathrm{Ca}$

2+ Handling

Arthur J. Cheng, Nicolas Place and Håkan

Westerblad
Effects of Exercise and Aging on Skeletal Muscle Giovanna Distefano and Bret $H$. Goodpaster

Muscle-Adipose Tissue Cross Talk Kristin I. Stanford and Laurie J. Goodyear

Performance Fatigability: Mechanisms and Task Specificity

Sandra K. Hunter

Adaptations to Endurance and Strength Training David C. Hughes, Stian Ellefsen and Keith Baar

The Bioenergetics of Exercise

$P$. Darrell Neufer

Effects of Exercise on Vascular Function,

Structure, and Health in Humans

Daniel J. Green and Kurt J. Smith

Control of Muscle Metabolism by the Mediator

Complex

Leonela Amoasii, Eric N. Olson and Rhonda

Bassel-Duby

Theoretical and Biological Evaluation of the Link between Low Exercise Capacity and Disease Risk Lauren Gerard Koch and Steven L. Britton

For additional articles in this collection, see http://perspectivesinmedicine.cshlp.org/cgi/collection/ 